\title{
An Automatic On-site Fire Ant Screening System
}

\author{
Sanqiang Zhao ${ }^{1,2}$, Yongsheng $\mathrm{Gao}^{2,1}$ \\ ${ }^{1}$ Queensland Research Laboratory, NICTA, Australia \\ ${ }^{2}$ Institute for Integrated and Intelligent Systems \\ Griffith University, Brisbane, Australia \\ \{s.zhao,yongsheng.gao\}@griffith.edu.au
}

\author{
Terry Caelli ${ }^{3}$, Fabian Bracco ${ }^{4}$ \\ ${ }^{3}$ Victoria Research Laboratory, NICTA, Australia \\ ${ }^{4}$ Griffith School of Engineering, Griffith University, \\ Brisbane, Australia \\ terry.caelli@nicta.com.au
}

\begin{abstract}
This paper proposes the first attempt for semiautomatic screening and identification of red imported fire ants (Solenopsis invicta) in Australia. As an exotic ant species to Australia, fire ants were imported from South America in 2001 and have since been regarded as dangerous pests that could severely damage the environment and many industries. We followed two of the three major identification keys defined by entomologists and proposed: 1) A fusion of two different image features (i.e., the perpendicular median intensity and the perpendicular width) for antenna segment detection; and 2) $\mathrm{A}$ weighted histogramming of micropattern features for petiole classification. Our experimental results show that automatic onsite fire ant screening is feasible and the proposed weighted histogramming of micropattern features performs better than the original micropattern representation.
\end{abstract}

Keywords—insect identification; fire ant; automatic screening; template matching

\section{INTRODUCTION}

As one of the world's most intractable invaders, the red imported fire ant was introduced to Australia in February 2001. They have since been regarded as dangerous imported pests that could spread to large areas of Australia, severely damaging the environment, livestock, agriculture, tourism, businesses and people's outdoor lifestyle [1]. In response, the Australian Government embarked on a $\$ 123 \mathrm{M}$ campaign to prevent the spread of these noxious pests. Commenced in September 2001, the National Red Imported Fire Ant Eradication Program [2], funded by the federal government and all Australian states and territories, built on an emergency response to locate and eradicate fire ants. The nationally coordinated program is managed by Biosecurity Queensland for the Department of Employment, Economic Development and Innovation.

The eradication program has made significant progress in eradicating fire ants from Australia. However, current implementations of the program are labor-intensive and timeconsuming. A typical scenario is that a resident identifies a suspected nest of fire ants and alerts Biosecurity Queensland, and then Biosecurity Queensland sends out a field inspector to collect and return samples to laboratories for identification. If a fire ant is confirmed, appropriate remedial action is prescribed to eradicate the outbreak. With most suspected outbreaks in remote areas, and specialist entomologists concentrated in cities, testing and identification can potentially be delayed, providing greater opportunity for fire ants to become established in an area and leading ultimately to environmental and industrial devastation. Moreover, human expertise that is required to correctly identify fire ants is very limited. For example, there are only around four full-time entomologists devoted to fire ant identification at Biosecurity Queensland Control Centre (BQCC). Therefore, constant monitoring, prompt detection and automatic identification of fire ants in situ play important roles for the control and eradication of fire ants across Australia.

For computerized insect identification, there are two broad categories, i.e., hierarchical top-down classifications using taxonomic keys, and case-by-case bottom-up searching using known species samples. The first category employs the existing keys or creates new keys to classify insects from the broadest levels (e.g., phylum) to the finest levels (e.g., species). This is the natural way used by human beings when doing insect classification without any target species. The difficulties are that the keys are very diverse from macro features such as wings and head to micro features such as hair and very small protrusion at the tail. It is therefore difficult to develop a universal system to cover all the keys. It is also difficult to automatically detect and locate the subtle keys which are challenges even for human experts. The advantages are the adaptiveness and flexibility. One can use this approach to classify to any level of taxonomy. An example of such system is Lucid [3], a package of commercial software tools designed to help users to perform identification and diagnostic tasks with flexible knowledge management. Despite the wide usage of the software, Lucid is a matrix-structured framework which requires professional taxonomists to manually define and prefeed the identification keys. In other words, it cannot be directly used by non-expert people without the prior knowledge input from professionals.

In the second category, image-based matching can be used to compare an input image with all of the images of known species based on pre-defined visual similarities. This approach is straight-forward to implement as existing computer vision and pattern recognition techniques can be applied to the insect identification problem. However, the whole image matching process is generally slow, because it may need to process up to millions of images. This approach also cannot provide generic information because there are no common images of an order or family of insects so that the only result is the finest level (mostly species). Two examples of such system are DAISY [4] and SPIDA [5]. In these systems, primitive image features such

This project was funded by Department of Agriculture, Fisheries and Forestry on behalf of Commonwealth Government of Australia and jointly conducted by NICTA and BQCC. 
as pixel-wise correlation and centroid, momentum are used to measure the similarity of images, and then nearest neighbor, neural network or support vector machine are used for classification. These systems can only operate on a small group of species, for instance, identification of 35 moth (sub-order) species [6]. The biggest number of species these systems can handle is up to 200 , with the performance at around $80 \%$.

This paper proposes the first prototype system of its kind for automatic screening and identification of red imported fire ants. The proposed system can be considered as a hybrid approach as discussed above. In the following, we provide a brief introduction of fire ants in Section II, a brief description of the prototype "In-Field Fire Ant Screening System" in Section III, the proposed algorithms used by the system in Section IV, the experimental results in Section V, and some discussions in Section VI.

\section{ABOUT FIRE ANTS}

\section{A. Size and Color}

The red imported fire ant (scientific name: Solenopsis invicta) originated from South America and was first imported to Brisbane, Queensland in February 2001. A typical fire ant is about 2 to $6 \mathrm{~mm}$ in length, with varied sizes within each nest (see Figure 1a). Its head and body are coppery-brown, and its abdomen is darker-brown (see Figure 1b). Figure 2 illustrates two line drawings which show the important taxonomic structures of ants from lateral and head views, respectively.

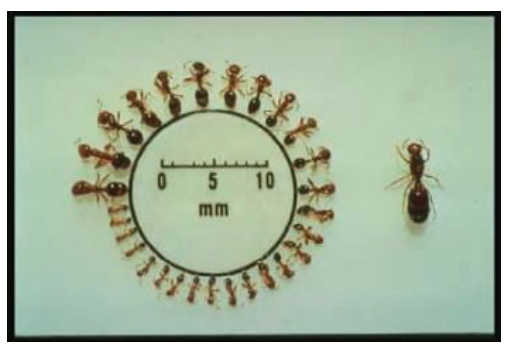

(a)

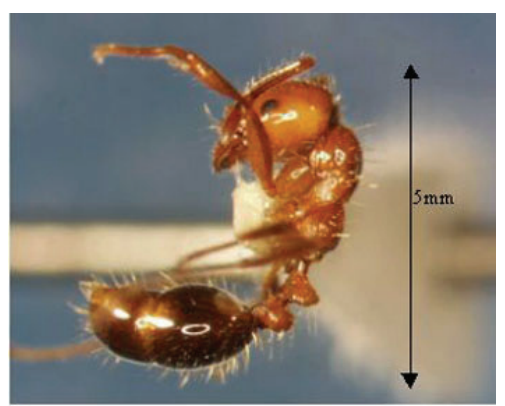

(b)

Figure 1. Fire ants (Photograph courtesy of Texas A\&M University). (a) Size of fire ants. (b) Color of fire ants.

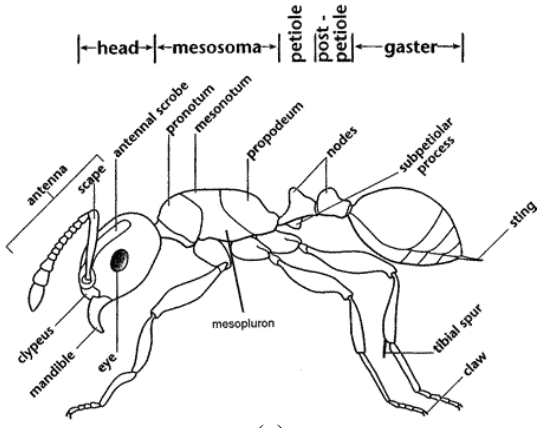

(a)

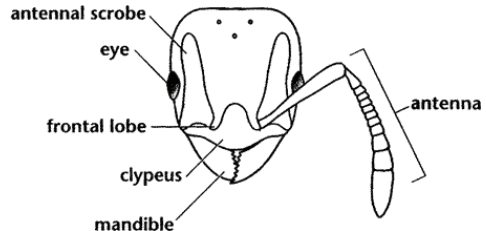

(b)

Figure 2. Taxonomic structures of ants [7]. (a) Lateral view. (b) Head view.

\section{B. Entomological Features}

Biosecurity Queensland entomologists use the following three major entomological keys to identify fire ants:

- There should be nine antenna segments excluding the base scape of the antenna (see Figure 3a).

- $\quad$ There should be two nodes (a petiole and post-petiole) at the petiole (see Figure 3b).

- There should be three teeth (one in the middle) on the clypeus (see Figure 3c).

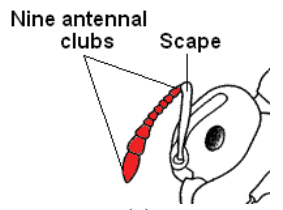

(a)

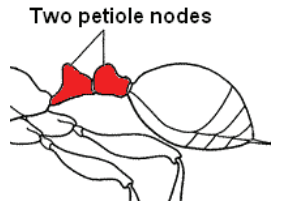

(b)

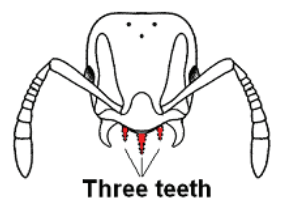

(c)

Figure 3. Entomological features used for fire ant identification. (a) Nine antenna segments. (b) Two petiole nodes. (c) Three clypeal teeth.

\section{AUTOMATIC IN-FiELD FIRE ANT SCREENING SYSTEM}

We have developed a prototype system, "In-Field Fire Ant Screening System", which is executable on Microsoft Windows platform with a low-cost USB-connected digital microscope Dino-Lite AM-413T [8]. Figure 4 illustrates a screenshot of the prototype system. At the current stage the system does not consider the head-view images due to the hardware limitation. 


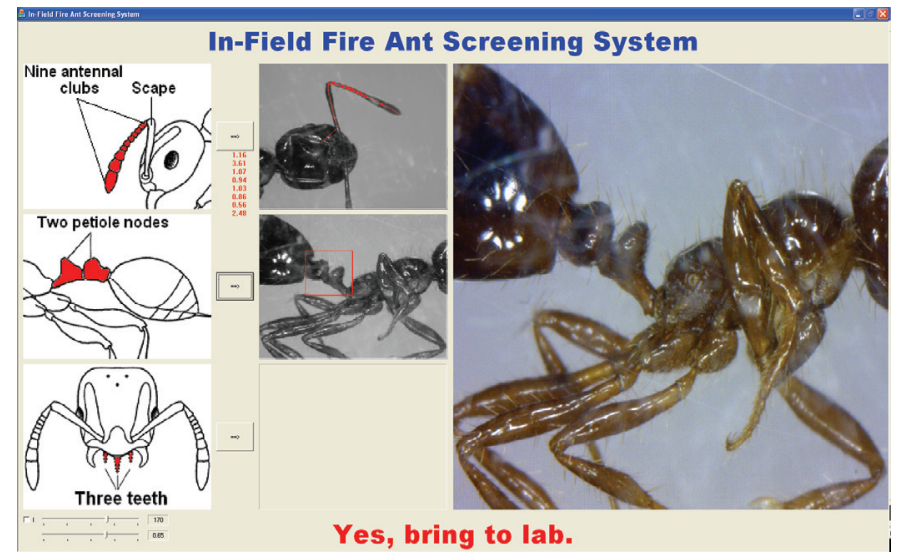

Figure 4. Screenshot of the prototype "In-Field Fire Ant Screening System".

\section{THE ALGORITHM}

We used a deterministic method to find the segments along the longest antenna on the antenna-view ant images. The template matching method was used to match the petiole-view images.

\section{A. Antenna Segment Detection}

Figure 5 illustrates the major image processing operations sequentially performed on the antenna-view ant images. With an input antenna-view image, pre-processing operations (histogram equalization and Gaussian smoothing) were first conducted to remove some interfering noise, followed by automatic thresholding to segment ant body from background. With the resulting binary image, two binary morphology operations, filling holes and skeletoning, were performed to consolidate the internal part of ant body and to skeletonize the ant antennas, respectively. Finally the longest terminating line was found from the skeleton image as the detected position of the ant antenna to be processed.

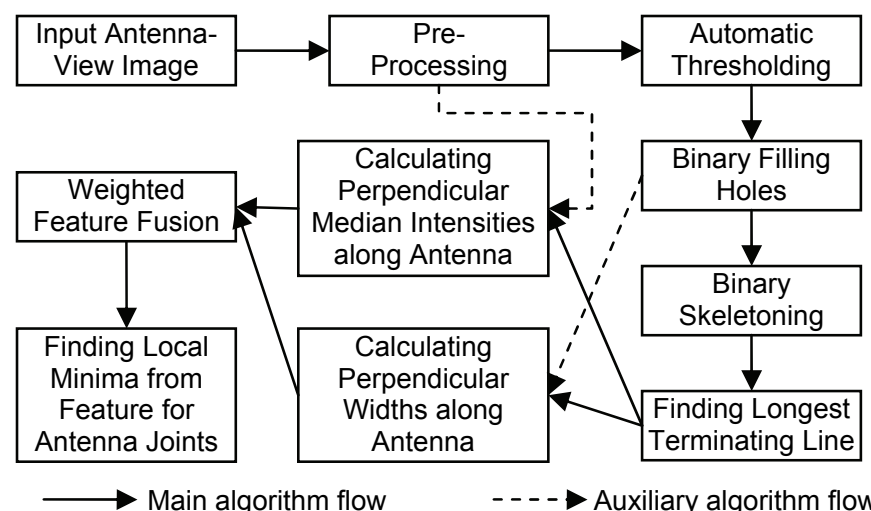

Figure 5. Flowchart of major operations of antenna segment detection.

We extracted two different features from each point of the detected antenna: the perpendicular median intensity and the perpendicular width. The first feature (see Figure 6a) is the median intensity extracted from the perpendicular pixels to the antenna at each point of the antenna. The second feature (see Figure $6 \mathrm{~b}$ ) is the width calculated from the perpendicular direction to the antenna at each point of the antenna. The final feature (see Figure 6c) was computed by a weighted fusion of the above two features $\left(F_{I}\right.$ and $\left.F_{W}\right)$ :

$$
F=F_{I}+\mu F_{W},
$$

where $\mu$ is the weight. In the system, the weight was heuristically selected as 3 . From the final feature, local minima (valley points in Figure 6c) were found as the detected connecting joints of antenna segments.

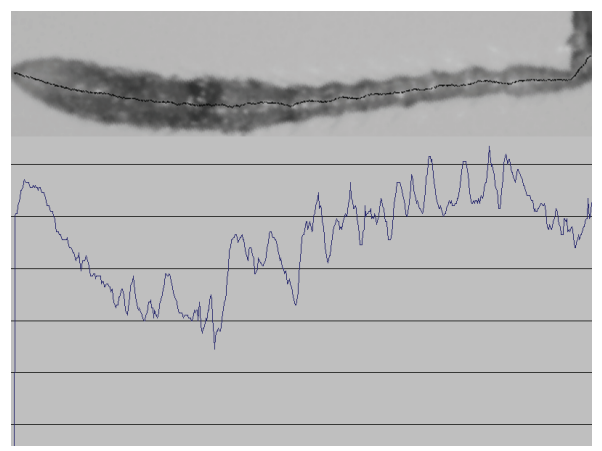

(a)

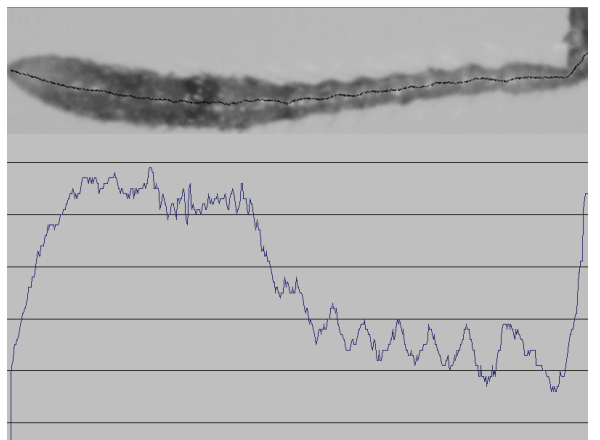

(b)

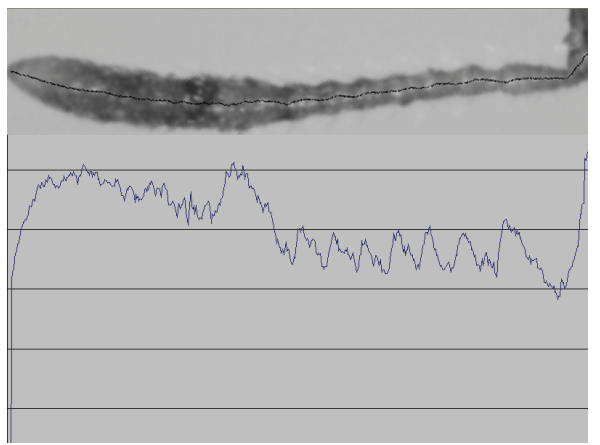

(c)

Figure 6. Features used for antenna segment detection. (a) The perpendicular median intensities. (b) The perpendicular widths. (c) Fusion features.

\section{B. Petiole Classification}

We manually cropped 16 fire ant petiole templates (Figure 7) from the training dataset for the use of petiole-view image processing. The templates are all $64 \times 64$ pixel sized and cover the whole in-plane rotation with about $45^{\circ}$ intervals. The sliding-window based template matching method was used to match the input image against each of the templates, with the similarity measured by the proposed weighted histogramming 
of Local Binary Pattern (LBP) micropattern features [9, 10]. Mathematically, LBP marks each pixel $I$ of an image as a decimal number $L B P^{P, R}(I)$, which is formed by thresholding $P$ equally spaced neighbor pixels $I^{p, R}(p=0, \cdots, P-1)$ on a circle of radius $R$ with the center pixel $I$ and concatenating the results binomially with factor $2^{p}$ :

$$
L B P^{P, R}=\sum_{p=0}^{P-1} s\left(I^{p, R}-I\right) 2^{p}, \quad s(x)=\left\{\begin{array}{ll}
1, & x \geq 0 \\
0, & x<0
\end{array} .\right.
$$

Let $\left\{h_{b}\right\}_{b=0 \cdots B}$ be the histogram of LBP micropatterns computed from a grey-level image region $\left\{I_{i}\right\}_{i=1 \cdots N}$ with $N$ pixels:

$$
h_{b}=\sum_{i=1}^{N} \delta\left(L B P_{i}^{P, R}-b\right) .
$$

The weight $\omega_{i}$ assigned to each pixel position is computed from the $Q$-value quantized average difference between each central pixel $I_{i}$ and its neighbors $I_{i}^{p, R}$ :

$$
\omega_{i}=\left\{\begin{array}{ll}
1, & d_{i} \in[0,256 / Q) \\
2, & d_{i} \in[256 \%, 256 \times 2 / Q) \\
\cdots, & \cdots \\
Q, & d_{i} \in[256 \times(Q-1) / Q, 256)
\end{array} \quad d_{i}=\frac{1}{P} \sum_{p=0}^{P-1}\left|I_{i}^{p, R}-I_{i}\right| .\right.
$$

The new weighted histogram $\left\{g_{b}\right\}_{b=0 \cdots B}$ is then defined by

$$
g_{b}=C \sum_{i=1}^{N} \omega_{i} \delta\left(L B P_{i}^{P, R}-b\right)
$$

with the normalization constant $C$ expressed as

$$
C=1 / \sum_{i=1}^{N} \omega_{i} .
$$

To preserve spatial information, both template images and sliding windows are divided into $4 \times 4$ non-overlapping rectangular sub-regions and represented with a spatial histogram which concatenates the histograms of all the subregions.

The scanning window with the maximum similarity on an input image is considered as the detected petiole position. When this maximum similarity value is above a pre-defined threshold, the detected petiole is considered as positive fire ant petiole. To cover petiole-size variation, the template matching was performed on three different downsized scales of input images $(22 \%, 20 \%, 18 \%$ resolution of original $1280 \times 1024$ images).

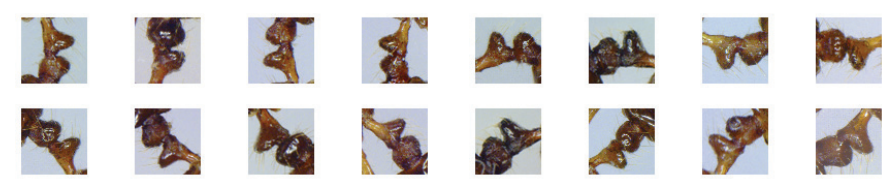

Figure 7. Example templates used for petiole classification.

\section{THE EXPERIMENTS}

Out prototype system has been evaluated on an ant image dataset captured by BQCC. The details of the dataset and the evaluation results are reported as follows.

\section{A. The BQCC Ant Dataset}

BQCC keeps a large repository of dead ant samples which include both fire ants (majority) and non-fire ants. The ant samples are first placed between two transparent and flat plastic plates, and then both the ant samples and plastic plates are placed on the desk top with a white background. The DinoLite AM-413T microscope is placed on the top of the upper plastic plate to take the pictures of ant samples. For each ant sample, multiple images from two different views (i.e., antenna view and petiole view) are captured. All the images are 24-bit color images with the resolution of $1280 \times 1024$ pixels. The ant images are named following the convention of Sol-i-001-01 (i.e. 3 characters for ant Genus; 1 character for ant Species; 3 digits for ant individual number; 2 digits for ant image number). Therefore, for all fire ant images, the filenames start

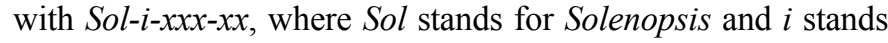
for invicta. For non-fire ant images, the filenames are different. For example, the filenames of Monomorium ant images start with Mon- $x-x x x-x x$. It should be noted that the last digit of an ant image filename indicates the capturing view. This digit can only be 1,2, 3 or 4, where 1 and 2 stand for antenna view and 3 and 4 stand for petiole view. Figure 8 displays some example images in the dataset.
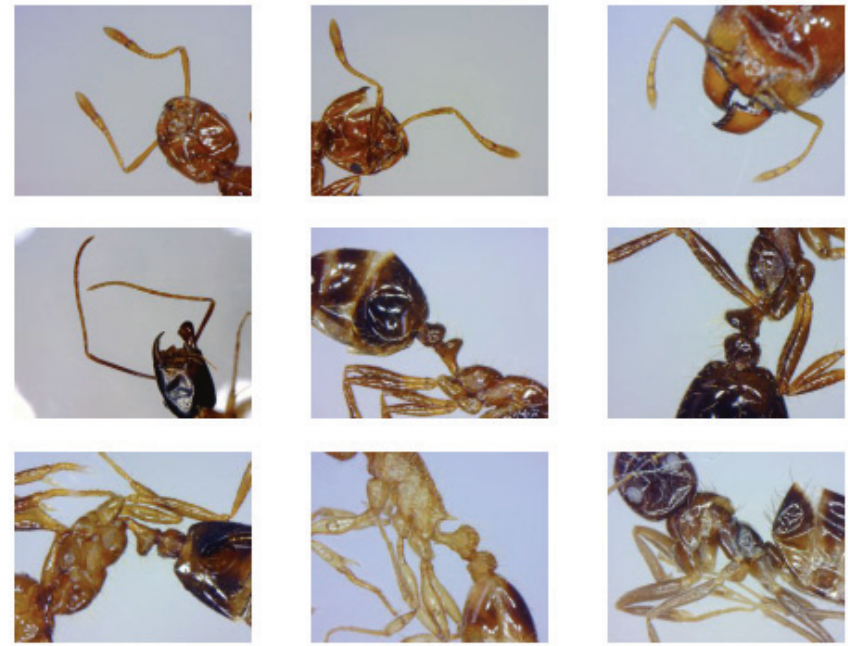

Figure 8. Example images in the BQCC ant dataset (first four images from antenna view and the rest from petiole view).

The BQCC ant dataset contains 1806 images, in which 1041 images are from antenna view and 765 images are from petiole view. For antenna view, 745 images are from fire ant samples and 296 images are from non-fire ant samples. For petiole view, 533 images are from fire ant samples and 232 images are from non-fire ant samples.

\section{B. The Experimental Results}

The prototype system was evaluated on the petiole-view images from the BQCC ant dataset using the LBP micropattern 
$L B P^{8,1}$. To compare the proposed weighted histogramming method of micropattern representation, comparative performances with both original and weighted histograms of LBP features have been reported. All the petiole-view images with two petiole nodes have been manually labeled the positions of the petioles (at the connecting joint of the two petiole nodes) as the ground truth.

Two statistic criteria are used to numerically measure the performance of the prototype system: Cascaded Correct Localization and Verification Rate (CCLVR) and False Alarm Rate (FAR). CCLVR is the percentage of fire ant images whose petioles are correctly detected (within 32-pixel distance away from the ground truth) and also correctly authenticated as fire ants by the system. FAR is the percentage of non-fire ant images which are incorrectly identified as fire ants. By changing the threshold in the system, a series of CCLVRs and FARs were obtained to draw a pseudo-ROC curve for evaluation of the system.

1) Experiment 1: To fairly evaluate the performance of the system, three experiments were set up. In Experiment 1, 533 fire ant images and 175 single-petiole-node non-fire ant images were used. To determine the quantization value of $Q$ in Equation (4), a set of experiments with different $Q$ was first conducted on Experiment 1. The recognition performances are plotted in Figure 9. The CCLVR reached optimal when $Q=16$. The parameter $Q=16$ was selected and used in the rest of the experiments. The comparative recognition performances of Experiment 1 are displayed in Figure 10. It can be observed from the figure that with the proposed weighted histogramming method, the system obtained $74.91 \%$ correct classification rate with no false alarms, and further increased to $86.52 \%$ correct classification rate at the cost of $17.61 \%$ false alarm rate, which is consistently about $5 \%$ higher than that of the original micropattern histogramming method.

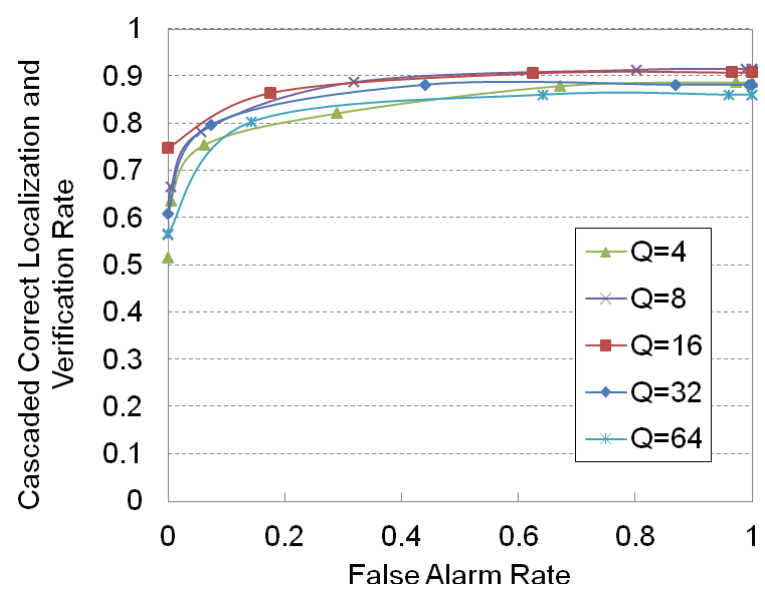

Figure 9. Recognition performances against different quantization values of $Q$ on Experiment 1 .

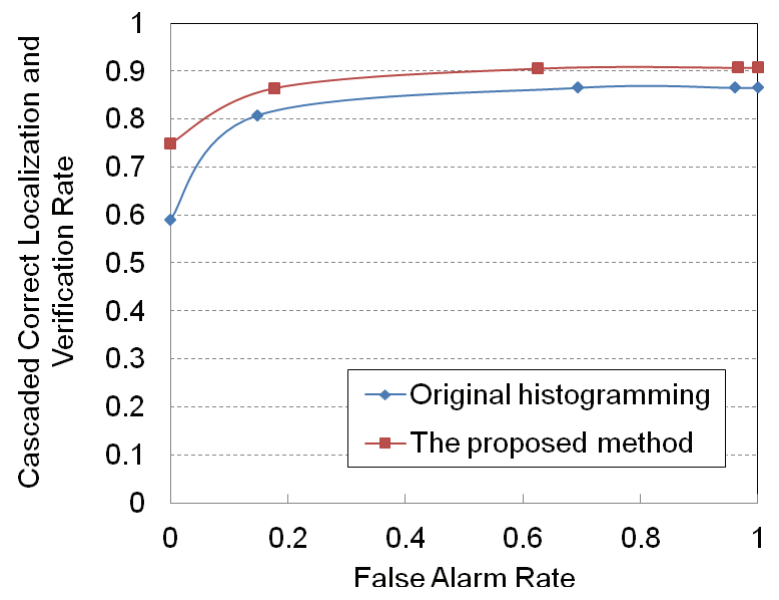

Figure 10. Comparative recognition performances on Experiment 1.

2) Experiment 2: In Experiment 2, 533 fire ant images and 232 non-fire ant images were used. Note the 232 non-fire ant images include both single-petiole-node images and twopetiole-node images. Because some two-petiole-node non-fire ant images are very similar to fire ant images, this experiment setting considerably increases the verification difficulty. The comparative experimental results are displayed in Figure 11. It can be observed from the figure that the system obtained only $27.15 \%$ correct classification rate with no false alarms, and further increased to $79.78 \%$ correct classification rate at the cost of $16.38 \%$ false alarm rate. This result is significantly worse than that of Experiment 1 but not surprising, because only based on the petiole-view images, even human could easily consider many two-petiole non-fire ant images as fire ant, not to mention the prototype system. It should be noted that the proposed method still performs about 5\% higher than the original micropattern histogramming method.

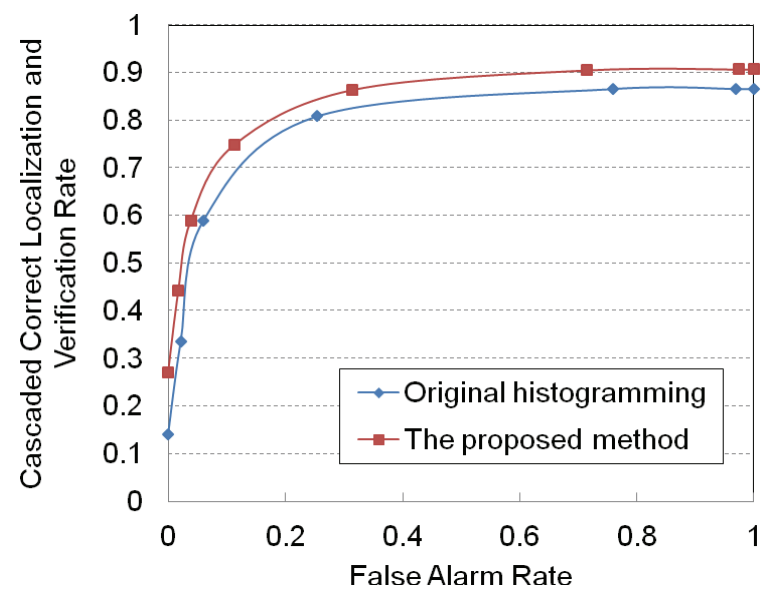

Figure 11. Comparative recognition performances on Experiment 2.

3) Experiment 3: In Experiment 3, 533 fire ant images and 232 non-fire ant images were used, which is same as Experiment 2. However, different from Experiment 2, the incorrectly recognized two-petiole-node non-fire ant images 
are intentionally counted as CCLVR instead of FAR. This is because for petiole-view images, the ability to distinguish two petioles from single petiole is adequate for a multi-key recognition task. The comparative experimental results are displayed in Figure 12. It can be observed from the figure that the system obtained $71.96 \%$ correct classification rate with no false alarms, and further increased to $85.30 \%$ correct classification rate at the cost of $17.61 \%$ false alarm rate. This result is similar to that of Experiment 1 and further proves the effectiveness of our prototype system. Note that the proposed method consistently performs better than the original micropattern histogramming method.

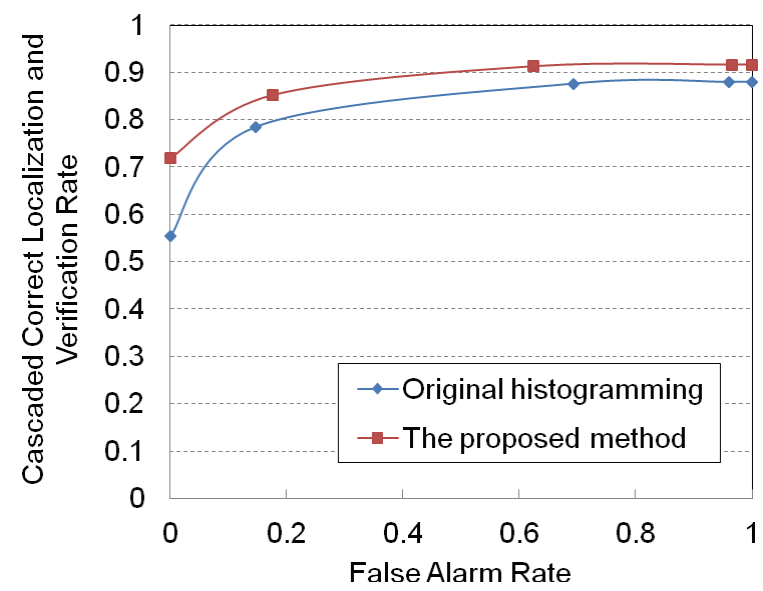

Figure 12. Comparative recognition performances on Experiment 3.

\section{CONCLUSIONS}

We developed a semi-automatic prototype system to assist fire ant screening and identification in Australia. This project is the first work of its kind for automatic fire ant screening using pattern recognition techniques. Our prototype system achieved over $70 \%$ correct petiole classification rate with no false alarms on the BQCC ant dataset. The experimental results reveal that using a single feature is insufficient to correctly classify fire ants to the level of human experts. Further research efforts are required to include all the three entomological keys towards robust fire ant recognition which is consistent to the human perception procedure of insect classification.

The prototype system was not evaluated on the antennaview images because although the proposed antenna segment detection method is working, counting the number of segments for antenna classification is very sensitive to noise and interference compared to petiole classification. Through our experiments we also proved that the proposed weighted histogramming of micropatterns performs better than the original micropattern representation.

\section{REFERENCES}

[1] S. Moloney, C. Vanderwoude: Red Imported Fire Ants: A threat to eastern Australia's wildlife? Ecological Management \& Restoration, 3(3): 167-175, 2002.

[2] NRIFAEP - National Red Imported Fire Ant Eradication Program, http://www.dpi.qld.gov.au/4790_4551.htm

[3] The Lucid Central - Home of the Lucid Family of Software, http://www.lucidcentral.com/

[4] K. Gaston, M. O'Neill: Automatic species identification: why not? Philosophical Transactions of the Royal Society B-Biological Sciences, 359(1444): 655-667, 2004.

[5] C. Wen, D.E. Guyer, W. Li: Automated insect classification with combined global and local features for orchard management, in Annual International Meeting of the American Society of Agricultural and Biological Engineers, 2009.

[6] M. Mayo, A.T. Watson: Automatic species identification of live moths, Knowledge-Based Systems, 20(2): 195-202, 2007.

[7] S.O. Shattuck: Australian ants: Their biology and identification, CSIRO Publishing, Collingwood, Australia, 1999.

[8] Dino-Lite Digital Microscope, http://www.dino-lite.com/

[9] T. Ojala, M. Pietikäinen, T. Mäenpää: Multiresolution gray-scale and rotation invariant texture classification with local binary patterns, IEEE Transactions on Pattern Analysis and Machine Intelligence, 24(7): 971987, 2002.

[10] S. Zhao, Y. Gao, B. Zhang: Sobel-LBP, IEEE International Conference on Image Processing (ICIP), pp. 2144-2147, 2008. 